\title{
Impaired Feedforward Control and Enhanced Feedback Control of Speech in Patients with Cerebellar Degeneration
}

\author{
Benjamin Parrell, ${ }^{1,2}$ Zarinah Agnew, ${ }^{3}$ Srikantan Nagarajan, ${ }^{4}$ John Houde, ${ }^{3}$ and Richard B. Ivry ${ }^{2}$ \\ ${ }^{1}$ Department of Linguistics and Cognitive Science, University of Delaware, Newark, Delaware 19716, ${ }^{2}$ Department of Psychology, University of California-Berkeley, \\ Berkeley, California 94720, and Departments of ${ }^{3}$ Otolaryngology and ${ }^{4}$ Radiology, University of California-San Francisco, San Francisco, California 94143
}

The cerebellum has been hypothesized to form a crucial part of the speech motor control network. Evidence for this comes from patients with cerebellar damage, who exhibit a variety of speech deficits, as well as imaging studies showing cerebellar activation during speech production in healthy individuals. To date, the precise role of the cerebellum in speech motor control remains unclear, as it has been implicated in both anticipatory (feedforward) and reactive (feedback) control. Here, we assess both anticipatory and reactive aspects of speech motor control, comparing the performance of patients with cerebellar degeneration and matched controls. Experiment 1 tested feedforward control by examining speech adaptation across trials in response to a consistent perturbation of auditory feedback. Experiment 2 tested feedback control, examining online corrections in response to inconsistent perturbations of auditory feedback. Both male and female patients and controls were tested. The patients were impaired in adapting their feedforward control system relative to controls, exhibiting an attenuated anticipatory response to the perturbation. In contrast, the patients produced even larger compensatory responses than controls, suggesting an increased reliance on sensory feedback to guide speech articulation in this population. Together, these results suggest that the cerebellum is crucial for maintaining accurate feedforward control of speech, but relatively uninvolved in feedback control.

Key words: ataxic dysarthria; cerebellum; feedback control; feedforward control; speech; speech motor control

Significance Statement

Speech motor control is a complex activity that is thought to rely on both predictive, feedforward control as well as reactive, feedback control. While the cerebellum has been shown to be part of the speech motor control network, its functional contribution to feedback and feedforward control remains controversial. Here, we use real-time auditory perturbations of speech to show that patients with cerebellar degeneration are impaired in adapting feedforward control of speech but retain the ability to make online feedback corrections; indeed, the patients show an increased sensitivity to feedback. These results indicate that the cerebellum forms a crucial part of the feedforward control system for speech but is not essential for online, feedback control.

\section{Introduction}

The cerebellum is associated with a variety of functions required for the coordination of movement, including sequencing, timing, motor programming, inverse modeling, and sensory prediction (Manto et al., 2012). In the speech domain, lesion and neuroim-

\footnotetext{
Received Oct. 31, 2016; revised July 25, 2017; accepted July 27, 2017.

Author contributions: B.P., Z.A., J.H., S.N., and R.B.I. designed research; B.P. performed research; B.P. analyzed data; B.P., J.H., S.N., and R.B.I. wrote the paper.

This work was supported by National Institutes of Health Grants F32 DC014211 to B.P., R01 NS092079 to R.B.I., R01 DC010145 to J.H. and S.N., and R01 DC013979 to J.H. and S.N., National Science Foundation BCS 1262297 to J.H. and S.N., and the Hearing Research Inc. to J.H. and S.N. We thank Adam Lammert for assistance with the latency estimation method.

The authors declare no competing financial interests.

Correspondence should be addressed to Dr. Benjamin Parrell, University of Wisconsin-Madison, Department of Communication Sciences and Disorders, 1975 Willow Drive, Madison, WI 53706. E-mail: bparrell@wisc.edu.

DOI:10.1523/JNEUROSCI.3363-16.2017

Copyright $\odot 2017$ the authors $\quad 0270-6474 / 17 / 379249-10 \$ 15.00 / 0$
}

aging studies have implicated the cerebellum in the speech motor control network (Bohland and Guenther, 2006; Ackermann et al., 2007; Ackermann, 2008; Ghosh et al., 2008). Although the nature of the functional contribution has received less attention than that observed in studies of limb or eye movement control, the cerebellum has been implicated in both feedforward and feedback control processes (Tourville and Guenther, 2011).

Given the rapidity of speech, control processes are assumed to rely principally on a feedforward system (Tourville and Guenther, 2011). This feedforward system is not fixed but is constantly updated based on past productions. This learning can be seen when speech is experimentally perturbed over an extended period: speakers adapt their output to oppose both auditory (Houde and Jordan, 1998; Purcell and Munhall, 2006a) and somatosensory (Tremblay et al., 2003; Lametti et al., 2012) perturbations. While neuropsychological studies have linked this type of adaptive learning with the cerebellum in motor domains such 
as reaching and locomotion (Taylor and Ivry, 2014), to date, no studies have examined speech adaptation in patients with cerebellar damage.

Speech motor control is not a purely feedforward process but also involves sensory feedback processing. This is clearly observed in experimental settings during which unpredictable altered feedback causes adjustment in online speech output. For example, when speech is artificially perturbed by altering pitch or vowel formants, speakers alter their production online to oppose these perturbations (Burnett et al., 1998; Purcell and Munhall, 2006b). Neuroimaging studies have shown increased cerebellar activation in response to both auditory (Tourville et al., 2008) and somatosensory (Golfinopoulos et al., 2011) perturbations of speech.

Although imaging studies have examined cerebellar activity in conditions designed to tax both feedforward and feedback aspects of speech motor control, it is difficult to interpret the functional role of the cerebellum from these results. For example, increased activation following perturbed speech may reflect recruitment of a feedback monitoring system or could reflect the fact that online adjustments based on sensory feedback increase the demands on motor output (Diedrichsen et al., 2005). Research in other motor domains suggests a greater role for the cerebellum in feedforward control, with a more modest contribution to feedback control. A consistent finding in the neuropsychological literature is that patients with cerebellar damage are impaired in adapting predictive, feedforward control of limb movements but that the cerebellum may not be essential for feedback control (Martin et al., 1996; Smith et al., 2000; Smith and Shadmehr, 2005; Morton and Bastian, 2006; Tseng et al., 2007).

The current paper examines the relative contribution of the cerebellum to feedback and feedforward control in speech. Given the evidence from gait and limb control, we hypothesize that the cerebellum is crucial for feedforward, but not feedback, control of speech. We test these hypotheses by assessing the ability of patients with cerebellar degeneration (CD) to alter their speech in response to perturbations of their auditory feedback (Houde and Jordan, 1998; Purcell and Munhall, 2006a, b). To assess feedforward control, we examine how the patients adapt their speech across trials when presented with a consistent perturbation of the sensory feedback. To assess feedback control, we examine online corrective responses within a trial in response to inconsistent perturbations.

Studying speech provides a new opportunity to assess hypothesized cerebellar function across motor domains. Moreover, speech provides a unique opportunity to study the feedback control system as the compensatory response to feedback errors is only partial in healthy speakers (Purcell and Munhall, 2006b), unlike the complete compensation seen in reaching or gait studies (Morton and Bastian, 2006; Tseng et al., 2007). This incomplete adaptation allows us to identify possible increases in the use of sensory feedback to guide ongoing productions.

\section{Materials and Methods \\ Participants}

Nineteen patients with CD (10 males) were tested (mean \pm SD age: $61.5 \pm 10.7$ years). These patients presented with heterogeneous diagnoses, including various types of spinocerebellar ataxia (SCA): SCA2 (2), SCA3 (2), SCA5 (1), SCA6 (2), SCA7 (1), SCA8 (2), and unknown/ idiopathic cerebellar atrophy (9). No CD patients reported any history of neurological damage or disorder apart from cerebellar atrophy. Fourteen controls (5 males) were recruited, matched in terms of age (65.1 \pm 7.7 years). Apart from typical high-frequency hearing loss associated with

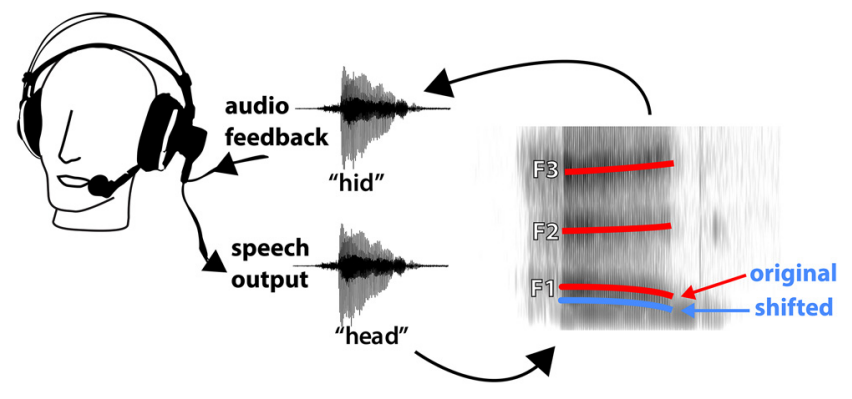

Figure 1. Schematic of auditory feedback manipulation process. The participant's speech is recorded via a head-mounted microphone and passed to the computer. The digital signal processing software estimates the vowel formants (F1-F3, red lines), introduces a desired shift (shown in blue as a negative displacement of F1), and resynthesizes the speech for playback to the participant via over-the-ear headphones. This process induces a change in the perceived vowel. In this example, the production of "head" would sound like "hid."

aging, none of the participants reported a history of speech or hearing problems.

The protocol was approved by the Institutional Review Board of the University of California, Berkeley.

\section{Apparatus}

Auditory feedback was altered using the Feedback Utility for Speech Production (FUSP) system, a digital signal processing system that can alter speech formants in real time (described in detail by Katseff et al., 2012). FUSP consists of a headset-mounted microphone, over-the-ear headphones, and a desktop PC. The participant's speech is recorded with the microphone and then passed to the computer for real-time processing (Fig. 1). Speech is repeatedly sampled in $3 \mathrm{~ms}$ windows or frames (32 samples at $11025 \mathrm{~Hz}$ ), and each frame is successively added to a running $36 \mathrm{~ms}$ (400 sample) buffer. For each resynthesized frame, vowel formants are estimated from this running buffer using linear predictive coding and shifted as specified by the experimental protocol (see below). The new formants are used to resynthesize the speech signal using a sinusoidal synthesis method (Quatieri and McAulay, 1986). This effectively introduces some smoothing into the resynthesized formants. The resynthesized speech is then played back to the participant over the headphones. The tight fit of the headphones, combined with relatively loud playback of the modified speech signal, serves to block transmission of external acoustic information. This system induces a total delay of $\sim 12 \mathrm{~ms}$ from utterance to feedback, which is imperceptible. The recordings from FUSP, in addition to being used for real-time resynthesis, are saved for offline behavioral analysis.

\section{Procedures}

Experiments 1 and 2 were completed by all participants in a single $45 \mathrm{~min}$ session. Participants first completed Experiment 1 and, following a short break, Experiment 2.

\section{Experiment 1}

Experiment 1 was designed to test feedforward control by examining speech adaptation across trials. To this end, FUSP was used to consistently perturb participants' production of the vowel $/ \varepsilon /$ (as in head) by reducing the first vowel formant (F1). In separate blocks of trials, this perturbation was introduced either in an abrupt or incremental manner. An overview of the experimental design is shown in Figure $2 a$.

Each trial began with a word presented on the computer screen that the participants were instructed to read in a normal voice. After $3.25 \mathrm{~s}$, the screen turned blank. The intertrial interval had a mean of $4 \mathrm{~s}$ (range 3.5-4.5 s) and ended with the reappearance of the word for the next trial.

The experiment began with an 80 trial practice block consisting of 8 repetitions each of 10 words: shed, said, led, wed, fed, pled, sled, sped, fled, and bled. The stimuli were presented in a random order. Productions were resynthesized through FUSP, but no perturbation was applied 
a

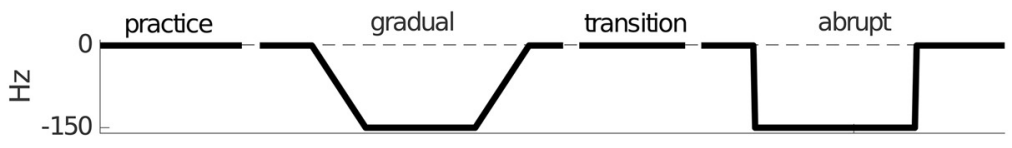

b

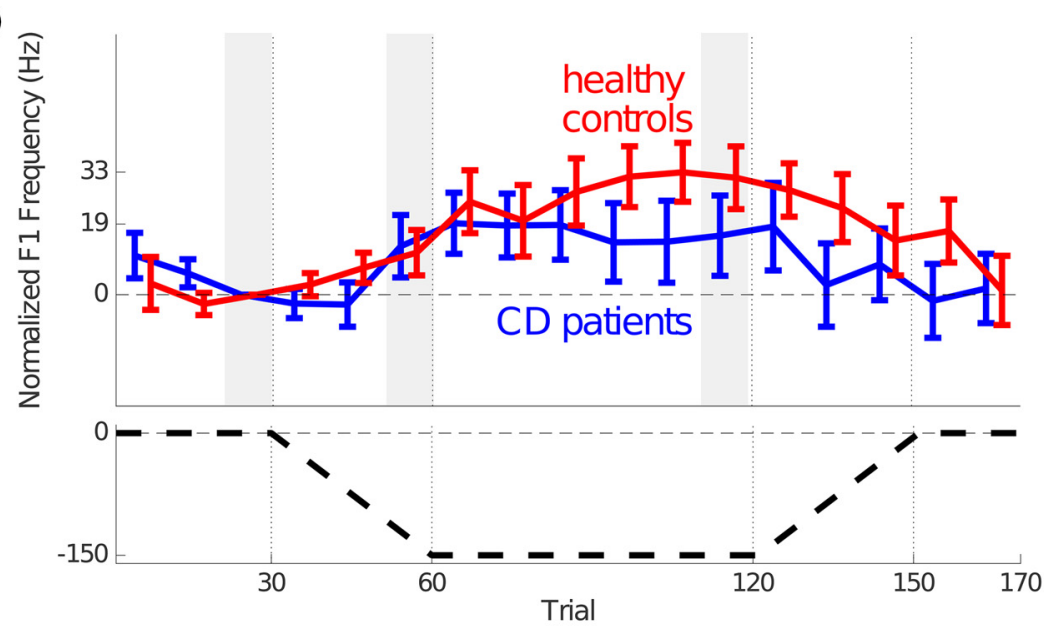

d

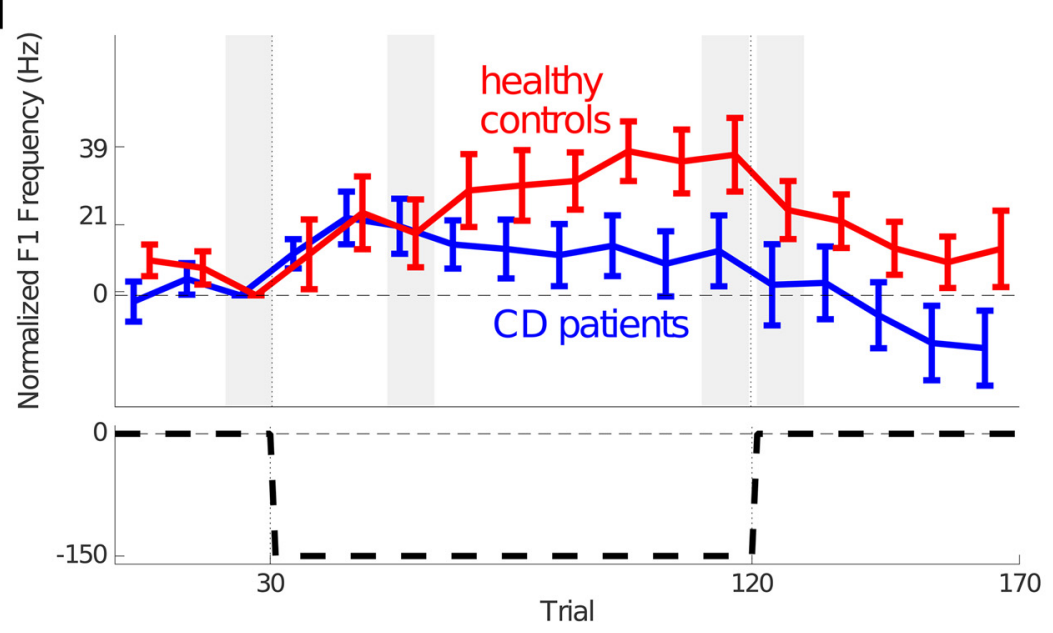

C

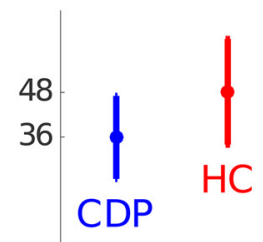

0

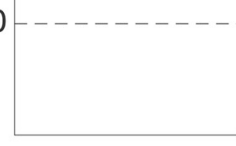

Catch Trials

e
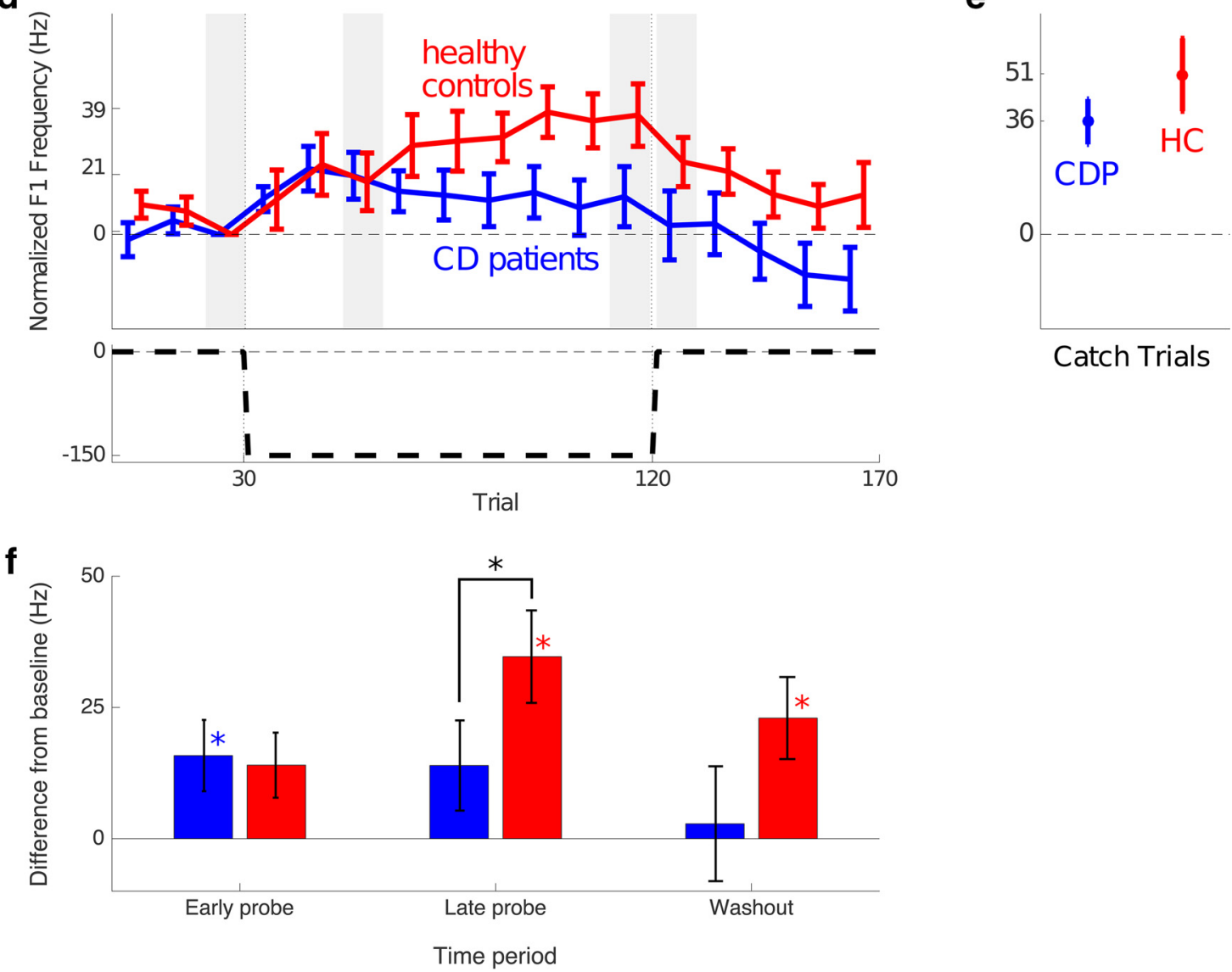

Figure 2. Experimental design and results from Experiment 1 on feedforward adaptation. $\boldsymbol{a}$, Schedule of perturbations in Experiment 1. Conditions of interest are the gradual and abrupt perturbations. $\boldsymbol{b}, \boldsymbol{d}$, Behavioral results from noncatch trials for gradual and abrupt perturbations, respectively. Productions are grouped into bins of 10. Error bars indicate SE. Light gray represents probes (baseline, early, late, washout). The perturbation schedule is shown below the behavioral results (black). There was no difference in results between the two conditions. $\boldsymbol{f}$, Control (red) and patient (blue) productions in the early probe, late probe, and washout phases, normalized to the baseline values. *Significant difference from baseline. $\boldsymbol{c}, \boldsymbol{e}$, Productions during the catch trials in the gradual and abrupt conditions, respectively. In these trials, loud speech-shaped noise was used to mask feedback from the participants' speech. On these trials, participants responded by dramatically increasing F1. This type of increase is typical in loud noise and makes it problematic to measure an adaptive response. Nonetheless, the trend in both cases is that CD patients produce a reduced increase in $\mathrm{F} 1$ compared with healthy controls. 
(veridical feedback). This block served to familiarize participants with the FUSP system before the start of the experimental blocks.

The main experiment consisted of two perturbation conditions (gradual and abrupt), separated by a 60 trial transition block. It has been hypothesized that CD patients may retain the ability to adapt the feedforward control system when the perturbation is introduced gradually, rather than in an abrupt manner (Criscimagna-Hemminger et al., 2010; Izawa et al., 2012). However, other studies have found reduced adaptive learning in $\mathrm{CD}$ patients regardless of how the perturbation is introduced (Gibo et al., 2013; Schlerf et al., 2013). In separate blocks, we use conditions in which an auditory perturbation is introduced gradually or abruptly to test possible effects of perturbation introduction on speech adaptation.

Two stimuli words were used, bed and head, with one word used for all trials in the gradual condition (baseline, perturbation, and washout blocks) and the other word used for all trials in the abrupt condition. The word-to-condition assignment was counterbalanced across participants. The use of different words for each condition should limit transfer between conditions (Rochet-Capellan et al., 2012).

The baseline block for both conditions consisted of 30 trials with veridical feedback. The two conditions differed in the perturbation block. In the gradual condition, the $\mathrm{F} 1$ perturbation was gradually introduced at $-5 \mathrm{~Hz}$ /trial over 30 trials. The maximal $-150 \mathrm{~Hz}$ perturbation was then maintained for 60 trials before being decreased by $5 \mathrm{~Hz} /$ trial over the final 30 trials of the block. A 20 trial washout block with veridical feedback followed the perturbation block. In the abrupt condition, the $-150 \mathrm{~Hz}$ F1 perturbation was introduced on the first trial of the perturbation block and remained present for all 90 trials of this block. This was followed by a 50 trial washout block with veridical feedback.

In sum, there were a total of 170 trials in each condition. The same order was used for all participants, with the gradual condition preceding the abrupt condition. By gradually increasing and decreasing the perturbation, we sought to keep participants unaware of the perturbation in the gradual condition. Removing the perturbation completely in the washout block would have introduced an abrupt change (from $-150 \mathrm{~Hz}$ perturbation of F1 to no perturbation). We were concerned that participants would notice this large change, and might attempt to explicitly compensate for it when tested in the abrupt condition. Similarly, if the abrupt condition was tested before the gradual condition, they might become sensitive to the perturbation and, should they use a strategy in the abrupt condition, redeploy that strategy in the gradual block if the difference between the expected and actual feedback became salient (Morehead et al., 2015). Short breaks were provided after the practice block, the abrupt condition, and the transition block.

In both conditions, 15 randomly selected trials during the hold phase (final 60 trials of the maximal perturbation in both conditions) were designated as catch trials. On these trials, participants heard speechshaped noise, played at a volume sufficient to mask the perception of their own speech (Villacorta et al., 2007). This effectively eliminated auditory feedback of speech. Performance on these catch trials was expected to reflect purely feedforward control. However, examination of the data revealed that all participants produced significantly greater F1 values during the catch trials compared with any other time during the experiment (Fig. $2 c, e$ ), an effect that is commonly observed when producing speech in loud noise (i.e., Lombard speech) (Summers et al., 1989). Performance on catch trials thus reflects the interaction of experimental-driven adaption and this additional factor. Because we did not include baseline catch trials, it is not possible to estimate the adaptation component; as such, we only provide a qualitative description of performance on these trials.

\section{Experiment 2}

Experiment 2 was designed to test the feedback response to perturbations within a single trial. On each trial, a single word was presented on the computer display. The words were drawn, at random, from the set: beck, bet, deck, debt, pet, and tech (Niziolek and Guenther, 2013). Participants were instructed to speak the word at a slightly slower rate than normal without artificially stretching out their production. This was done so that the participants would have sufficient time to generate within-trial cor- rective responses. This protocol is based on previous work, which has shown that corrections occur $\sim 150-200 \mathrm{~ms}$ from the acoustic onset of the vowel (Tourville et al., 2008; Cai et al., 2012; Niziolek and Guenther, 2013). To facilitate productions at the desired rate, feedback was provided after each trial. If the produced word was shorter than $400 \mathrm{~ms}$, a yellow circle appeared on the screen below the stimulus word; if the production was longer than $400 \mathrm{~ms}$, a green circle appeared in the same location. If the participant produced an excessively long utterance (generally over $1 \mathrm{~s}$ ), the experimenter paused the experiment and reminded the participant to speak in a normal, albeit slow, manner.

The experimental block consisted of 170 trials. The first 10 trials were produced with veridical feedback and used to train the participants to speak at the desired rate. On a random subset of the subsequent 160 trials, F1 was perturbed either up (shift-up condition) or down (shift-down condition) by $150 \mathrm{~Hz}$. Perturbations could only occur, at most, on two sequential trials. Our original plan was to have perturbations on 80 of the 160 trials (40 upward and 40 downward). However, due to a coding error in the stimulus presentation software, the actual number of perturbations was lower for some of the participants. For 4 participants (all CD patients), upward perturbations were made on 20 trials (downward on 40 trials); for 16 other participants ( $7 \mathrm{CD}, 9$ controls), downward perturbations were made on 20 trials (upward on 40 trials). The analyses showed no difference in the feedback response of participants who received an asymmetric perturbation schedule. As such, all participants were included in the analyses.

We recognize that, with the random perturbations used in Experiment 2, between-trial adaptation may continue to operate, even if it is no longer functionally useful (Diedrichsen et al., 2005). However, given the random order of the perturbation direction and the constraint that perturbations could occur on only two consecutive trials, we expect any possible trial-to-trial learning in the current experiment will not substantially alter participant behavior. Moreover, given that adaptation effects in speech production show very limited generalization, performance should be minimally affected by adaptation because we use a large set of words (Rochet-Capellan et al., 2012).

All participants were tested on Experiment 2 after completing Experiment 1 , with the full experimental session lasting $\sim 45 \mathrm{~min}$. These conditions were not counterbalanced for the same reason the abrupt condition always followed the gradual condition in Experiment 1 . Namely, we were concerned that the abrupt changes in the size of the auditory perturbation from trial to trial in Experiment 2 might cause participants to become sensitive to the perturbations and that this might lead to a strategic adjustment in behavior. Given the perturbation could either entail an increase or decrease of $150 \mathrm{~Hz}$ in $\mathrm{F} 1$, the difference across trials could be $300 \mathrm{~Hz}$, assuming the produced $\mathrm{F} 1$ value was constant. Additionally, we found in testing that many participants continued to produce the slow speech used for Experiment 2 in Experiment 1 when the order was reversed despite instructions to speak at a normal rate. Using the fixed order avoided this issue.

\section{Behavioral measures and statistical analyses}

In-house software was used to automatically track F1. The vowel for each production was detected based on a subject-specific amplitude threshold. The duration of the vowel was recorded from the time the amplitude crossed this threshold until the time the amplitude subsequently fell below this threshold. When this resulted in very short $(<150 \mathrm{~ms})$ or very long $(>500 \mathrm{~ms}$ ) vowels, these trials were hand-checked for accuracy. Formants were estimated at each time point in the vowel using linear predictive coding, with the number of linear predictive coding parameters and the amount of preemphasis set for each participant.

Trials where F1 showed obvious signs of incorrect tracking (e.g., large jumps, values $<250 \mathrm{~Hz}$, values $>1000 \mathrm{~Hz}$, or durations $<150 \mathrm{~ms}$ ) were corrected by hand. We excluded from the final analysis trials in which it was not possible to accurately track F1 or where participants did not produce the correct response (no response, coughing, etc.). This resulted in the exclusion of 84 trials (1.3\%) in Experiment 1 and 45 trials (1.4\%) in Experiment 2. 


\section{Experiment 1}

The main dependent variable was the average produced F1. This value was obtained by averaging the $\mathrm{F} 1$ values over the window $50-100 \mathrm{~ms}$ after the onset of the vowel. This window was chosen to (1) avoid large effects of the initial consonant in the formant trajectory and (2) restrict the analysis to a period of time before there could be feedback-based adjustments to the production (Niziolek and Guenther, 2013). Given these criteria, we assume that changes in $\mathrm{F} 1$ across the perturbation and washout blocks reflect adaptation from the feedforward control system.

The F1 values for each trial were normalized by subtracting, on a trial-by trial basis, the mean F1 produced during the last 10 trials of the baseline phase. Given that every speaker has a unique F1 range for a given vowel, this normalization method allows us to compare the adaptive response across speakers. We also used a second normalization procedure that included the entire baseline phase, here dividing the value for each trial by the baseline mean to calculate a percentage change, rather than absolute change. The results were consistent with both normalization methods. As such, we report only the absolute change values.

Late adaptation was measured by comparing the mean F1 values over the last 10 trials of the hold phase to the last 10 trials of the baseline phase (late probe). In addition, a measure of early adaptation (early probe, the mean F1 value over Trials 51-60) was included to compare the rate of adaptation between the two groups. Productions in the washout phase (the mean of the first 10 trials after the removal of the perturbation) were also measured. The washout phase was only included for the abrupt condition, as the perturbation was ramped down in the gradual condition, preventing measurement of an aftereffect.

A linear mixed-effects model was built using the lme4 package in $\mathrm{R}$ (Bates et al., 2014). The model included three fixed factors: group (CD vs Control), probe time (Baseline, Early, Late, Washout), and condition (Gradual vs Abrupt) with random intercepts for each subject. Statistical significance was assessed with the lmerTest package (Kuznetsova et al., 2016), and nonsignificant factors were excluded from the final models. Post hoc tests with Tukey corrections were conducted with lsmeans (Lenth, 2016). The reported degrees of freedom for these models represent approximated values of the effective (pooled) degrees of freedom (Satterwaithe approximation for lmerTest and Kenwood-Roger for lsmeans).

\section{Experiment 2}

The main dependent variable was the trajectory of F1 during the initial $400 \mathrm{~ms}$ of the vowel. This duration was chosen based on a preliminary analysis showing that most trials $(\sim 90 \%)$ had a vowel duration of at least $400 \mathrm{~ms}$ (our duration feedback was designed to encourage participants to produce vowels that were at least $400 \mathrm{~ms}$ in duration). The mean trajectory for all unperturbed productions was calculated for each stimulus word. This baseline F1 trajectory was subtracted from the F1 trajectory of all perturbed trials on a word-specific basis to generate difference trajectories (i.e., deviation from the mean) for each perturbed trial.

To measure feedback responses, we calculated the magnitude and latency of the compensatory response from the difference trajectories of each perturbation trial. Differences in the magnitude of the compensatory response between patients and controls were determined by constructing, at each time point, a linear mixed-effects model with group (CD patient vs healthy control) and perturbation direction (up vs down), and their interaction, as fixed factors and random intercepts for participants. Overall differences in the final magnitude of the response were calculated over the time period from 300 to $400 \mathrm{~ms}$ after vowel onset. Means were calculated in this window for the up, down, and unperturbed conditions for each subject. These by-subject means were then used for statistical testing.

Latencies were calculated using a spline fitting method (Cai et al., 2012). Each perturbed trial was fit with a two-piece linear spline such that:

$$
C(t)=\left\{\begin{array}{c}
x_{0}, t<L \\
x_{0}+b(t-L), t \geq L
\end{array}\right.
$$

Where $x_{0}$ is the baseline F1 before the onset response, $L$ is the response latency, and $b$ is the slope of the response from time $L$ to the end of the analysis window. These parameters were fit to each trial using the function fmincon in MATLAB. A lower bound for $L$ was set to $50 \mathrm{~ms}$ based on the shortest latencies reported in past work (Purcell and Munhall, 2006a; Cai et al., 2012; Niziolek and Guenther, 2013). As previous work has shown that only a subset of trials in similar tasks elicit a compensatory response, we identified compensating trials as those trials where the identified latency $(L)$ occurred within the $400 \mathrm{~ms}$ analysis window and the slope of the response $(b)$ opposed the perturbation. Only this subset of trials was used for estimating latencies.

Two additional tests were conducted to investigate possible contributions in Experiment 2 of (1) trial-to-trial, feedforward learning and (2) strategy use. To test for the presence of feedforward learning, we examined the F1 value produced in the first $50 \mathrm{~ms}$ of the vowel, comparing trials following an upward perturbation, a downward perturbation, or no perturbation. If trial-to-trial learning were occurring, we would expect that this measure would show an inverse relationship with the preceding perturbation (e.g., participants would shift F1 downward after an upward perturbation if they were learning to adapt their feedforward plan). To examine strategy use, we reasoned that strategic responses would only develop after exposure to the perturbations. To test this, we compared the F1 values from the first and second halves of the perturbation phase (Trials 11-170). An increase in the response to the perturbation in the second half would be consistent with the implementation of an explicit strategy.

All statistical testing was conducted using linear mixed-effects model with group, perturbation direction, and their interaction as fixed factors with random intercepts for participants as in Experiment 1.

\section{Results}

\section{Experiment 1: feedforward control of speech}

Experiment 1 examined across-trial adaptation in CD patients and controls to consistent perturbations of F1. In two separate conditions, this perturbation was introduced either gradually or abruptly. Figure 2 presents the group-averaged F1 data over the course of the abrupt and gradual conditions. Linear, mixedeffects models were used to test for main effects of group (patients vs controls), probe time (baseline, early probe, late probe, washout), and condition (gradual vs abrupt) as well as the interactions between these factors. There was no difference between the two conditions $\left(F_{(1,186)}=1.3, p=0.26\right)$, and the condition $\times$ probe time interaction was not reliable $\left(F_{(2,186)}=0.2, p=0.81\right)$. Moreover, there was no interaction between condition and group $\left(F_{(1,186)}=0.2, p=0.64\right)$ or a three-way interaction of condition, probe time, and group $\left(F_{(2,186)}=0.2, p=0.80\right)$. These factors were excluded from the final model.

Both CD patients and controls were able to adapt their productions to oppose the perturbation, as indicated by a main effect of time $\left(F_{(3,192)}=10.3, p=3 \mathrm{e}-6\right)$. Although there was no overall main effect of group $\left(F_{(1,32)}=1.6, p=0.21\right)$, the patients and controls showed different patterns of adaptation, indicated by a significant interaction between group and time $\left(F_{(3,192)}=3.1\right.$, $p=0.02)$.

Post hoc tests were conducted to examine the two-way interaction between group and probe time. In the early probe, both groups showed similar amounts of adaptation, with patients having a mean shift of $15.8 \mathrm{~Hz}\left(t_{(192)}=2.8, p=0.03\right)$ and controls having a mean shift of $14.0 \mathrm{~Hz}\left(t_{(192)}=2.1, p=0.15\right)$, although the control effect was not significant when correcting for multiple comparisons. When compared directly, there was no difference between the two groups at the early probe $\left(t_{(66)}=0.20, p=0.84\right)$.

The performance of the two groups diverged with extended exposure to the perturbation. The controls continued to adapt such that, by the late probe (last 10 trials of the hold phase), the total shift in F1 reached a mean of $34.7 \mathrm{~Hz}$. This value was significantly larger than that observed during the early probe $t_{(192)}=$ 
$3.1, p=0.01)$. In contrast, the CD patients showed no significant difference between the early and late probes (mean late probe $=$ $\left.13.9 \mathrm{~Hz} ; t_{(192)}=0.3, p=0.98\right)$. In a direct comparison of the two groups at the late probe, the degree of adaptation for the control participants was significantly greater than that observed for the CD patients $\left(t_{(66)}=2.25, p=0.03\right)$.

This difference persisted in the washout phase, with only the controls showing a response different from baseline (mean shift $=22.9 \mathrm{~Hz}, t_{(192)}=2.8, p=0.03$ ). The CD patients did not show any difference between the washout and baseline blocks (mean shift $\left.=2.8 \mathrm{~Hz}, t_{(192)}=0.40, p=0.98\right)$. These results indicate that only the controls, but not CD patients, showed a persistent aftereffect of learning once the perturbation was removed. For the CD patients, the small aftereffect was not reliable when the data were averaged over 10 trials.

The catch trials show the same general pattern as found in the hold phase (Fig. $2 c, e$ ). Both the CD patients and controls show productions above baseline values (although some of this increase is likely due to general effects of speaking in loud noise and not specific to adaptation). This increase is reduced in the patients compared with the controls in both the gradual and abrupt conditions. The magnitude of the differences between groups $(\sim 15 \mathrm{~Hz})$ is similar to the differences seen in the noncatch trials during the hold phase.

Overall, the results indicate that the control participants adapted to the constant perturbation to a greater degree than the $\mathrm{CD}$ patients. This effect was evident in the larger change in F1 for the controls at the late adaptation probe and by the presence of a significant aftereffect for this group in the washout phase. A similar pattern has been observed in prior work with CD patients in reaching (Schlerf et al., 2013). It remains unclear whether this specificity reflects a dissociation between processes involved in the initial and late stages of learning or whether measures of performance are more sensitive in the latter due to the fact that performance has stabilized.

\section{Experiment 2: feedback control of speech}

Experiment 2 examined the online, within-trial compensatory response of $\mathrm{CD}$ patients and controls to unexpected perturbations. F1 was randomly perturbed, either up or down, on a subset of the trials. These perturbation trials were intermixed with control trials in which no perturbation was applied. The results reveal that both the CD patients and control speakers showed compensatory responses in both the shift-up and shift-down conditions. The participants produced formant trajectories that served to compensate for the perturbation, with F1 moving in the opposite direction of the perturbation over time (Fig. 3).

A compensatory response could be identified on $\sim 70 \%$ of the trials across all groups and conditions. There were no differences between groups in the frequency of their responses (patients: $71.5 \%$; controls: $\left.71.3 \%, F_{(1,31)}=0.3, p=0.59\right)$. Neither was there a difference based on perturbation direction (up: 70.7\%; down: $\left.71.6 \%, F_{(1,31)}=0.2, p=0.68\right)$ nor an interaction between these two factors $\left(F_{(1,31)}=0.8, p=0.36\right)$. The overall rate of response is consistent with that found for healthy speakers in previous work (Burnett et al., 1998), although this seems to depend on how response rate is calculated (Cai et al., 2012).

We focused on a window, 300-400 ms after the vowel onset, at which the magnitude of this compensatory response was greatest (Fig. $3 b$ ). Both CD patients and controls showed a compensatory response, indicated by a main effect of perturbation condition $\left(F_{(2,62)}=19.9, p=2 \mathrm{e}-7\right)$. Of greatest interest, the CD patients showed a larger response than controls $\left(F_{(1,62)}=5.0, p=\right.$
$0.03)$. There was no interaction between group and perturbation direction $\left(F_{(2,62)}=1.4, p=0.25\right)$. Responses in both the upward and downward perturbation conditions were significantly different from baseline (upward: mean $=-17.7 \mathrm{~Hz}, t_{(62)}=6.0, p=$ 0.00001 ; downward: mean $\left.=8.9 \mathrm{~Hz}, t_{(62)}=3.0, p=0.01\right)$. Additionally, the response to the upward perturbation was significantly larger than the response to the downward perturbation $\left(t_{(62)}=3.3, p=0.004\right)$.

The results from the entire time course of the response are consistent with the maximum compensation results (Fig. $3 a$ ). Here, too, the CD patients showed a larger compensatory response than the controls. This was a consistent trend across all time points in both conditions and reached significance from 275 to $320 \mathrm{~ms}$ after vowel onset (main effect of group, dark gray bars, $p<0.05$ ). Between 330 and $350 \mathrm{~ms}$, this enhanced response in CD patients was statistically significant only in the shift-up condition (interaction, black bars, $p<0.05$ ). There was also a significant effect of direction, such that responses in the shift-up condition were larger than in the shift-down condition from $200 \mathrm{~ms}$ on (main effect of condition, light gray bars, $p<0.05$ ).

The compensatory response, when present, began $\sim 150 \mathrm{~ms}$ after vowel onset (Fig. $3 d$ ). There was no difference between patients $(147 \mathrm{~ms})$ and controls $(152 \mathrm{~ms})$ on this measure $\left(F_{(1,31)}=\right.$ $0.5, p=0.46)$, nor were there differences in latency between the up $(144 \mathrm{~ms})$ and the down $(153 \mathrm{~ms})$ conditions $\left(F_{(1,1544)}=\right.$ $2.4, p=0.12)$. There was no interaction between the factors $\left(F_{(1,1544)}=0.01, p=0.91\right)$.

We conducted two additional analyses to explore other processes that might contribute to the performance differences between the groups. First, we tested for the presence of feedforward learning. If trial-to-trial learning were occurring, we would expect that the initial response on any given trial would show an inverse relationship with the preceding perturbation (e.g., participants would shift F1 downward after an upward perturbation if they were learning to adapt their feedforward plan). When analyzing the mean $\mathrm{F} 1$ value during the first $50 \mathrm{~ms}$ of articulation, no significant effect of perturbation direction $\left(F_{(2,62)}=1.0, p=\right.$ $0.38)$, patient group $\left(F_{(1,31)}=0.0002, p=0.99\right)$, or their interaction $\left(F_{(2,62)}=0.3, p=0.75\right)$ was observed. This suggests that there is little feedforward learning in Experiment 2 and that the patients and controls do not differ in this regard.

Second, we considered whether the participants modified their behavior over the course of the experiment, perhaps developing a strategic response to the perturbations. To test this hypothesis, we divided the perturbation block (Trials 11-170) into two halves. We found no difference in behavior between the first and second halves, with the mean adjustment (combined over groups) identical in the two halves $\left(15 \mathrm{~Hz}, F_{(1,154)}=0.005, p=\right.$ 0.95 ). This factor did not interact with participant group (patient vs control, $F_{(1,154)}=0.2, p=0.16$ ), perturbation direction (up vs down, $\left.F_{(2,154)}=1.2, p=0.16\right)$, or the combination of these factors $\left(F_{(2,154)}=0.3, p=0.72\right)$. These results indicate that the participants in both groups showed a consistent response to the perturbations throughout the experiment, and suggest that the participants did not adopt a strategy in an attempt to improve their performance.

To examine whether there was any relationship between the magnitude of compensation and adaptation, correlations were calculated between the average adaptive, feedforward response during the end of the hold phase for Experiment 1 (across both abrupt and gradual conditions), and the average compensatory, feedback response during the last $100 \mathrm{~ms}$ of the response for 

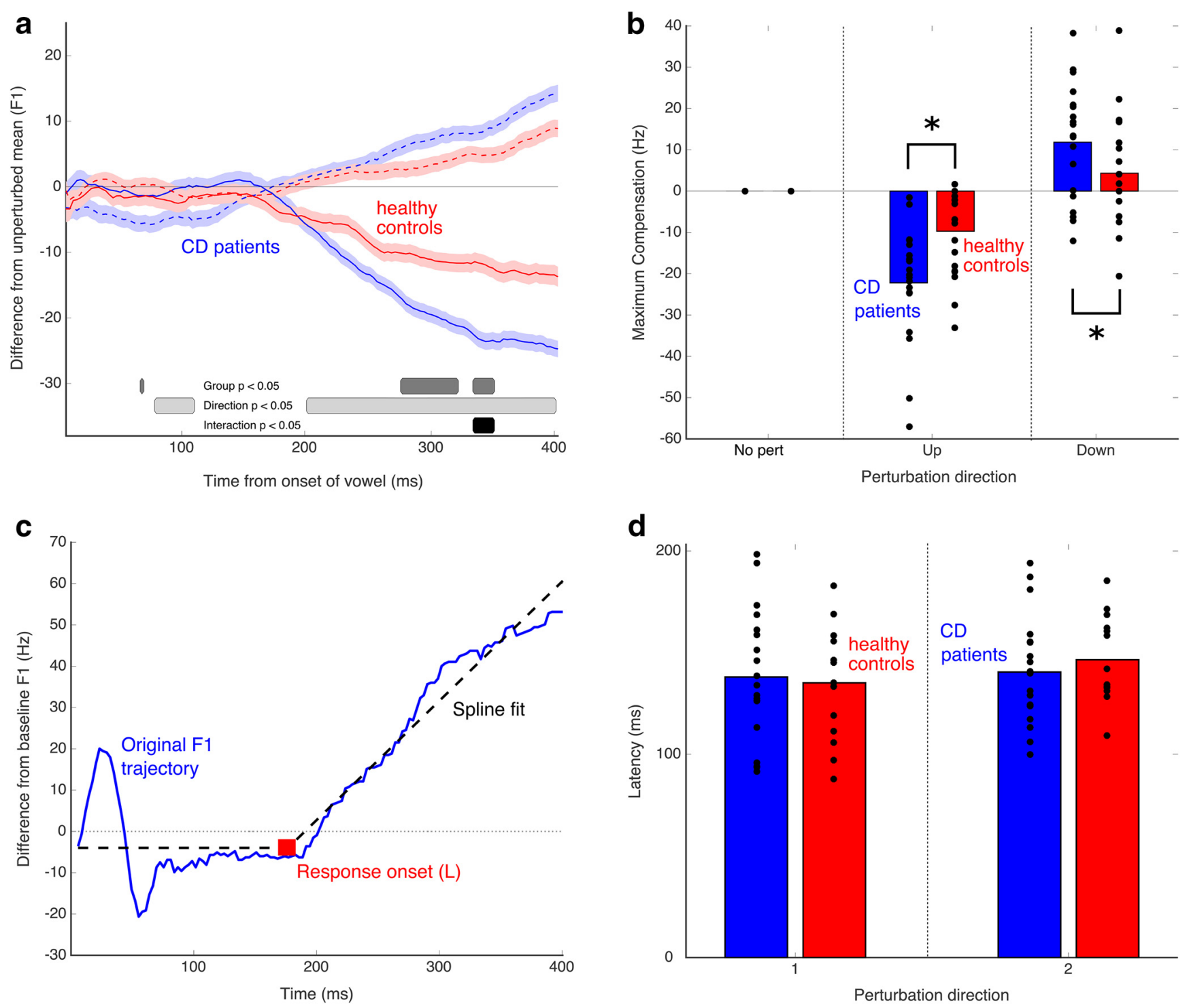

Figure 3. Results from Experiment 2 on feedback compensation. $\boldsymbol{a}$, Response of healthy controls (red) and CD patients (blue) to unexpected upward ( $150 \mathrm{~Hz}$, solid) and downward ( $-150 \mathrm{~Hz}$, dotted) perturbations of F1. The functions represent differences on perturbation trials, relative to baseline, shown over time averaged across participants. Shading represents $\mathrm{SE}$. $\boldsymbol{b}$, Maximum response (average from 300 to $400 \mathrm{~ms}$ after vowel onset) for the unperturbed, shift-up, and shift-down conditions. Means are shown along with individual responses (black circles). $c$, Example of the spline-fitting method used to calculate response latencies. $\boldsymbol{d}$, Mean latencies and individual responses. There were no differences between groups or perturbation directions. *Significant difference from baseline.

Experiment 2 (across both upward and downward perturbations). Correlations were calculated separately for CD patients and controls. No significant relationship was found for either CD patients $(r=-0.02, p=0.95)$ or for control participants $(r=$ $0.30, p=0.29)$.

\section{Discussion}

This paper reports two studies designed to test the hypothesis that the integrity of the cerebellum is essential for predictive, feedforward control of speech, but not essential for reactive, feedback control. We manipulated the auditory feedback that the participants received while speaking, shifting F1 in real time to alter the acoustic qualities of the perceived vowel. When the perturbation was consistent across trials, patients with CD showed reduced adaptation, consistent with a deficit in feedforward control. In contrast, the same patients showed a heightened reactive response in response to unpredictable perturbations of formant feedback, indicative of an intact, or perhaps hypersensitive, feedback system.

\section{Feedforward control}

In the first experiment, we introduced a consistent perturbation of F1 over a large number of trials. Speech adaptation was significantly impaired in the CD patients relative to controls. Whereas controls showed a large change in motor output when the perturbation was present and a significant aftereffect when the perturbation was removed, the patients showed a reduced shift under perturbation and minimal aftereffect. These results provide a novel demonstration of the critical role of the cerebellum in feedforward speech motor control and converge with similar observations in other motor domains (Shadmehr et al., 2010).

The CD patients exhibited a similar deficit when the perturbation was introduced in a gradual manner or imposed in an abrupt manner. The difference between these two conditions 
and, in particular, the relative involvement of the cerebellum, has been a point of debate in the literature. Whereas the results of some studies suggest a greater impairment with abrupt perturbations (Criscimagna-Hemminger et al., 2010; Izawa et al., 2012), others have also observed similar impairments with the two types of perturbations (Gibo et al., 2013; Schlerf et al., 2013). There are many differences between the two conditions; for example, with an abrupt perturbation, the initial error is larger and participants are more likely to be aware of the altered feedback, potentially engaging strategic processes to compensate for the perturbation (Taylor and Ivry, 2012; Taylor et al., 2014).

Most participants in speech perturbation studies are unaware of the perturbation. Even in cases where people report an awareness of some change, they are generally unable to specify exactly what has been altered. Moreover, there is no behavioral difference between aware and naive participants (Niziolek and Guenther, 2013). This suggests that, even if participants are aware of the perturbation, they would be unable to adopt a conscious strategy to alter their behavior. In this way, speech perturbation studies provide an excellent avenue for studying feedforward adaptation without interference from a strategic component.

The auditory feedback manipulation used here introduces a sensory prediction error, a mismatch between the expected and actual sensory consequences of an action. The cerebellum is postulated to be the locus for generating these expectations (Wolpert et al., 1998; Shadmehr and Krakauer, 2008). Importantly, sensory prediction errors have been shown to underlie sensorimotor adaptation of feedforward control (Tseng et al., 2007). Although our results suggest that updating of feedforward control is impaired in CD patients, we have not directly shown that feedforward control, in and of itself, is affected. However, it follows that an impaired inability to learn from sensory errors would cause problems in feedforward control itself, as movement planning would be reliant on miscalibrated and imprecise forward models (Tseng et al., 2007). Thus, the imprecise consonants and vowels characteristic of CD patients' speech are also consistent with an impairment in feedforward control.

\section{Feedback control}

We investigated online feedback control by examining participants' response to unpredictable F1 perturbations in a second experiment. The direction of the perturbation was randomized (upward, downward, or no perturbation) across trials.

The CD patients retained the ability to make appropriate feedback-based compensatory responses to the unexpected perturbations. This result is consistent with the hypothesis that feedback control in speech is not crucially dependent on the cerebellum, and is especially striking when contrasted with the impaired feedforward control observed in Experiment 1. Although the cerebellum appears in some models of feedback control in speech (Tourville and Guenther, 2011), our results are in accord with past work in other motor domains, where patients with cerebellar pathology were found to retain the ability to use sensory feedback to correct ongoing movements (Smith et al., 2000; Bastian, 2006; Tseng et al., 2007).

Interestingly, the CD patients in our study showed a larger compensatory response than the control participants. An enhanced feedback response has not been reported in other domains. It may be that the enhanced response following cerebellar damage is unique to speech. Alternatively, it may be that speech is particularly useful for examining the range of feedback control given that feedback-based compensation typically serves to counter only a portion of the imposed perturbation in speech (Purcell and Munhall, 2006b), whereas in other motor domains, such as reaching, feedback can be used to ensure that the target is fully attained (Tseng et al., 2007). The reason that compensation (and adaptation) to auditory perturbations in speech is incomplete is not clear, although refined methods have shown that adaptation in reaching may also be more limited than often reported (Bond and Taylor, 2015; Morehead et al., 2017). Possible reasons include principally reliance on feedforward control in speech, relative to other domains (Tourville and Guenther, 2011), competition with somatosensory feedback (Lametti et al., 2012; Mitsuya et al., 2015), or relatively broad and categorical targets for vowels (Niziolek and Guenther, 2013).

Regardless of the source, the incomplete compensation found in healthy speakers allowed us to observe that the CD patients produced a stronger response to adjust an ongoing movement. While in the current task, the increased use of feedback for online control resulted in a larger task-relevant correction, similar increases in other motor tasks may negatively impact performance or lead to unstable movements. For example, reliance on sensory feedback in a reaching task may result in target overshoot and intention tremor (Day et al., 1998).

The corrective feedback response was larger for the shift-up, compared with the shift-down, manipulation in both the patients and controls, a result that we had not predicted. Previous studies have shown that compensation effects are similar regardless of the direction of the perturbation (e.g., Purcell and Munhall, 2006b; Cai et al., 2012), although direct comparisons are usually not reported. There is one study showing a similar asymmetry as observed in the present study with the / $\varepsilon$ / vowel (Mitsuya et al., 2015). Thus, it may be that the asymmetry reflects some intrinsic difference in the sensitivity to up and down perturbations, at least in this context, either at the perceptual or motor end. Alternatively, the asymmetry in our study may result from the fact that our participants performed Experiment 2 immediately after Experiment 1 , and thus had previous exposure to the down-shifted, but not up-shifted, auditory feedback. This hypothesis suggests that the feedback response may be modulated by previously experienced errors. This possibility will be important to follow-up on in future work, including testing the hypothesis that error experience might not only affect feedforward control (Herzfeld et al., 2014) but could also influence the dynamics of feedback control (Wei and Körding, 2009). Interestingly, any changes to feedback control that might have been induced by previous exposure to a particular perturbation were similar in controls and patients given that the asymmetry was similar for both groups.

\section{Interaction between feedforward and feedback control}

There are different ways to interpret the finding of increased feedback responses in the CD patients. One hypothesis is that the enhanced feedback response reflects a shift in the dynamic balance between feedback and feedforward control: damage to the feedforward system automatically shifts the balance toward the feedback control system. Recent work on adaptation in reaching to visuomotor perturbations has shown that performance changes are a composite of multiple processes, including explicit re-aiming and implicit adaptation, and that these processes trade off with one another (Taylor et al., 2014). Although responses to speech perturbations may not entail these same processes, there may be an intrinsic trade-off between feedback and feedforward control. Alternatively, the increased use of feedback control in CD patients could be a learned compensatory mechanism for impaired feedforward control. Disruption in feedforward control 
would cause greater instability in motor production and might lead to greater reliance on an intact feedback system to ensure that motor output achieves the desired communicative goal.

The enhanced feedback response observed in the CD patients argues against a critical role for the cerebellum in feedback control. However, functional imaging studies have shown increased cerebellar activation when participants make online corrections for unexpected perturbations during speech (Tourville et al., 2008; Golfinopoulos et al., 2011). How can we reconcile the patient behavior and brain activity in healthy speakers?

Two (nonexclusive) hypotheses seem viable here. First, the motor output is likely greater on trials requiring online corrections. Diedrichsen et al. (2005) have shown that increases in the BOLD signal in various brain regions associated with reaching errors are related to the increased motor output in response to these errors. Although it is more difficult to quantify motor output in speech compared with reaching, it is reasonable to assume that motor commands are more complex when a corrective movement is required. Second, the increased cerebellar activity seen following speech perturbations may not reflect online reactive control, but rather recruitment of an error-based learning process (Wolpert et al., 1998; Shadmehr and Krakauer, 2008). In this view, cerebellar neural activity in response to sensorimotor perturbations is part of the feedforward learning process (updating a forward model) but not feedback control per se. Such a process would operate even when an induced perturbation is random; thus, the trial-to-trial corrections are not functional. The current results showing an impairment in feedforward adaptation with sparing of feedback control are consistent with this idea.

\section{References}

Ackermann H (2008) Cerebellar contributions to speech production and speech perception: psycholinguistic and neurobiological perspectives. Trends Neurosci 31:265-272. CrossRef Medline

Ackermann H, Mathiak K, Riecker A (2007) The contribution of the cerebellum to speech production and speech perception: clinical and functional imaging data. Cerebellum 6:202-213. CrossRef Medline

Bastian AJ (2006) Learning to predict the future: the cerebellum adapts feedforward movement control. Curr Opin Neurobiol 16:645-649. CrossRef Medline

Bates D, Maechler M, Bolker B, Walker S (2014) lme4: linear mixed-effects models using Eigen and S4. R package version 1.1-12.

Bohland JW, Guenther FH (2006) An fMRI investigation of syllable sequence production. Neuroimage 32:821-841. CrossRef Medline

Bond KM, Taylor JA (2015) Flexible explicit but rigid implicit learning in a visuomotor adaptation task. J Neurophysiol 113:3836-3849. CrossRef Medline

Burnett TA, Freedland MB, Larson CR, Hain TC (1998) Voice F0 responses to manipulations in pitch feedback. J Acoust Soc Am 103:3153-3161. CrossRef Medline

Cai S, Beal DS, Ghosh SS, Tiede MK, Guenther FH, Perkell JS (2012) Weak responses to auditory feedback perturbation during articulation in persons who stutter: evidence for abnormal auditory-motor transformation. PLoS One 7:e41830. CrossRef Medline

Criscimagna-Hemminger SE, Bastian AJ, Shadmehr R (2010) Size of error affects cerebellar contributions to motor learning. J Neurophysiol 103: 2275-2284. CrossRef Medline

Day BL, Thompson PD, Harding AE, Marsden CD (1998) Influence of vision on upper limb reaching movements in patients with cerebellar ataxia. Brain 121:357-372. CrossRef Medline

Diedrichsen J, Hashambhoy Y, Rane T, Shadmehr R (2005) Neural correlates of reach errors. J Neurosci 25:9919-9931. CrossRef Medline

Ghosh SS, Tourville JA, Guenther FH (2008) A neuroimaging study of pre- motor lateralization and cerebellar involvement in the production of phonemes and syllables. J Speech Lang Hear Res 51:1183-1202. CrossRef Medline

Gibo TL, Criscimagna-Hemminger SE, Okamura AM, Bastian AJ (2013) Cerebellar motor learning: are environment dynamics more important than error size? J Neurophysiol 110:322-333. CrossRef Medline

Golfinopoulos E, Tourville JA, Bohland JW, Ghosh SS, Nieto-Castanon A, Guenther FH (2011) fMRI investigation of unexpected somatosensory feedback perturbation during speech. Neuroimage 55:13241338. CrossRef Medline

Herzfeld DJ, Vaswani PA, Marko MK, Shadmehr R (2014) A memory of errors in sensorimotor learning. Science 345:1349-1353. CrossRef Medline

Houde JF, Jordan MI (1998) Sensorimotor adaptation in speech production. Science 279:1213-1216. CrossRef Medline

Izawa J, Criscimagna-Hemminger SE, Shadmehr R (2012) Cerebellar contributions to reach adaptation and learning sensory consequences of action. J Neurosci 32:4230-4239. CrossRef Medline

Katseff S, Houde J, Johnson K (2012) Partial compensation for altered auditory feedback: a tradeoff with somatosensory feedback? Lang Speech 55:295-308. CrossRef Medline

Kuznetsova A, Brockhoff P, Christensen RHB (2016) lmerTest: tests in linear mixed effects models. R package version 2.0-30.

Lametti DR, Nasir SM, Ostry DJ (2012) Sensory preference in speech production revealed by simultaneous alteration of auditory and somatosensory feedback. J Neurosci 32:9351-9358. CrossRef Medline

Lenth, R (2016) Least-squares means. R package version 2.25.

Manto M, Bower JM, Conforto AB, Delgado-García JM, da Guarda SN, Gerwig M, Habas C, Hagura N, Ivry RB, Mariën P, Molinari M, Naito E, Nowak DA, Oulad Ben Taib N, Pelisson D, Tesche CD, Tilikete C, Timmann D (2012) Consensus paper: roles of the cerebellum in motor control-the diversity of ideas on cerebellar involvement in movement. Cerebellum 11:457-487. CrossRef Medline

Martin TA, Keating JG, Goodkin HP, Bastian AJ, Thach WT (1996) Throwing while looking through prisms: I. Focal olivocerebellar lesions impair adaptation. Brain 119:1183-1198. CrossRef Medline

Mitsuya T, MacDonald EN, Munhall KG, Purcell DW (2015) Formant compensation for auditory feedback with English vowels. J Acoust Soc Am 138:413-424. CrossRef Medline

Morehead JR, Qasim SE, Crossley MJ, Ivry R (2015) Savings upon re-aiming in visuomotor adaptation. J Neurosci 35:14386-14396. CrossRef Medline

Morehead JR, Taylor JA, Parvin DE, Ivry RB (2017) Characteristics of implicit sensorimotor adaptation revealed by task-irrelevant clamped feedback. J Cogn Neurosci 29:1061-1074. CrossRef Medline

Morton SM, Bastian AJ (2006) Cerebellar contributions to locomotor adaptations during splitbelt treadmill walking. J Neurosci 26:9107-9116. CrossRef Medline

Niziolek CA, Guenther FH (2013) Vowel category boundaries enhance cortical and behavioral responses to speech feedback alterations. J Neurosci 33:12090-12098. CrossRef Medline

Purcell DW, Munhall KG (2006a) Adaptive control of vowel formant frequency: evidence from real-time formant manipulation. J Acoust Soc Am 120:966-977. CrossRef Medline

Purcell DW, Munhall KG (2006b) Compensation following real-time manipulation of formants in isolated vowels. J Acoust Soc Am 119:22882297. CrossRef Medline

Quatieri TF, McAulay RJ (1986) Speech transformations based on a sinusoidal representation. Acoust Speech Signal Process IEEE Trans on 34: 1449-1464. CrossRef

Rochet-Capellan A, Richer L, Ostry DJ (2012) Nonhomogeneous transfer reveals specificity in speech motor learning. J Neurophysiol 107:17111717. CrossRef Medline

Schlerf JE, Xu J, Klemfuss NM, Griffiths TL, Ivry RB (2013) Individuals with cerebellar degeneration show similar adaptation deficits with large and small visuomotor errors. J Neurophysiol 109:1164-1173. CrossRef Medline

Shadmehr R, Krakauer JW (2008) A computational neuroanatomy for motor control. Exp Brain Res 185:359-381. CrossRef Medline

Shadmehr R, Smith MA, Krakauer JW (2010) Error correction, sensory prediction, and adaptation in motor control. Annu Rev Neurosci 33:89-108. CrossRef Medline 
Smith A, Johnson M, McGillem C, Goffman L (2000) On the assessment of stability and patterning of speech movements. J Speech Lang Hear Res 43:277-286. CrossRef Medline

Smith MA, Shadmehr R (2005) Intact ability to learn internal models of arm dynamics in Huntington's disease but not cerebellar degeneration. J Neurophysiol 93:2809-2821. CrossRef Medline

Summers WV, Johnson K, Pisoni DB, Bernacki RH (1989) An addendum to "Effects of noise on speech production: acoustic and perceptual analyses" [J Acoust Soc Am 84:917-928 (1988) ]. J Acoust Soc Am 86:1717-1721. CrossRef Medline

Taylor JA, Ivry RB (2012) The role of strategies in motor learning. Ann N Y Acad Sci 1251:1-12. CrossRef Medline

Taylor JA, Ivry RB (2014) Cerebellar and prefrontal cortex contributions to adaptation, strategies, and reinforcement learning. Prog Brain Res 210: 217-253. CrossRef Medline

Taylor JA, Krakauer JW, Ivry RB (2014) Explicit and implicit contributions to learning in a sensorimotor adaptation task. J Neurosci 34:3023-3032. CrossRef Medline
Tourville JA, Guenther FH (2011) The DIVA model: a neural theory of speech acquisition and production. Lang Cogn Process 26:952-981. CrossRef Medline

Tourville JA, Reilly KJ, Guenther FH (2008) Neural mechanisms underlying auditory feedback control of speech. Neuroimage 39:1429-1443. CrossRef Medline

Tremblay S, Shiller DM, Ostry DJ (2003) Somatosensory basis of speech production. Nature 423:866-869. CrossRef Medline

Tseng YW, Diedrichsen J, Krakauer JW, Shadmehr R, Bastian AJ (2007) Sensory prediction errors drive cerebellum-dependent adaptation of reaching. J Neurophysiol 98:54-62. CrossRef Medline

Villacorta VM, Perkell JS, Guenther FH (2007) Sensorimotor adaptation to feedback perturbations of vowel acoustics and its relation to perception. J Acoust Soc Am 122:2306-2319. CrossRef Medline

Wei K, Körding K (2009) Relevance of error: what drives motor adaptation? J Neurophysiol 101:655-664. CrossRef Medline

Wolpert DM, Miall RC, Kawato M (1998) Internal models in the cerebellum. Trends Cogn Sci 2:338-347. CrossRef Medline 